\title{
A Comprehensive Multiphase NMR Probehead with Reduced Radiofrequency Heating Improves the Analysis of Living Organisms and Heat Sensitive Samples
}

Paris Ning ${ }^{\mathrm{a}}$, Daniel Lane ${ }^{\mathrm{a}}$, Rajshree Ghosh Biswas ${ }^{\mathrm{a}}$, Ronald Soong ${ }^{\mathrm{a}}$, Daniel Schmidig ${ }^{\mathrm{b}}$, Thomas Frei ${ }^{\mathrm{b}}$, Peter De Castro ${ }^{b}$, Ivan Kovacevic ${ }^{b}$, Stephan Graf ${ }^{b}$, Sebastian Wegnerc ${ }^{c}$, Falko Busse ${ }^{c}$, Jochem Struppe ${ }^{d}$, Michael Fey $^{\mathrm{d}}$, Henry J. Stronks ${ }^{\mathrm{e}}$, Martine Monette ${ }^{\mathrm{e}}$, Myrna J. Simpsona, and André J. Simpson*a

a. Environmental NMR Centre, Department of Physical and Environmental Science, University of Toronto Scarborough, Toronto, ON, Canada, M1C 1A4

b. Bruker BioSpin AG, 8117 Fällanden, Switzerland

c. Bruker BioSpin Corp., Billerica, Massachusetts, USA, 01821-3991

d. Bruker Biospin GmbH, Silberstreifen 4, 76287 Rheinstetten, Germany

e. $\quad$ Bruker Ltd., Milton, ON, Canada, L9T 6P4

\section{Supporting Information}

Number of Pages: 5

Number of Figures: 3 


\section{H. azteca Survival Testing}

To test the survivorship under high-power decoupling conditions, each shrimp was subjected to a 12-hour long CP experiment with $100 \mathrm{kHz}$ decoupling bandwidth on both probes. The rotor was set at the magic angle, but not spun. This was to eliminate additional stress from spinning, making it easier to specifically focus on the impact of stress from heating. The rotor was ejected every hour for visual inspection to determine the survivorship of each shrimp. Shrimps that had survived the full 12-hour duration were unloaded from the rotor to a beaker with culture media to further confirm their survival. A plot between survivorship and experiment length was produced for both probes (see Fig. 1A main paper).

To further confirm the death arises solely from high-power decoupling induced RF sample heating, two additional sets of experiments were acquired. The first test subjected each living shrimp to $2500 \mathrm{~Hz}$ MAS rate for 12 hours $^{1}$ along with a CP pulse but with decoupling power turned off (with other parameters identical to the reference conditions). All shrimps survived the $12 \mathrm{~h}$ test on both probes.

A final experiment subjected living shrimp to hourly injecting/ejecting of the rotor to mimic the hourly observation in the survival test. $2500 \mathrm{~Hz}$ MAS, a CP pulse and no decoupling, similar to the first test, were also applied. This test was designed to rule out differences in, for example, the rotor ejection between the probes. Note the top transfer line (same used for both probes) has a small piece of foam inserted designed to reduce the sudden impact of the rotor hitting the top of the tube to reduce impact stress on the organisms. This test lasted 12 hours and all shrimps survived. The results of these two tests confirm that mortality of shrimps is only observed when decoupling is applied, and indeed, the stress, leading to death, arises from the high decoupling power. 


\section{Calibration Curve of KBr Solution Chemical Shift with Temperature}

Calibration of temperature vs ${ }^{79} \mathrm{Br}$ chemical shift was performed using a single $90^{\circ}$ pulse for excitation on the ${ }^{79} \mathrm{Br}$ channel. The temperature steps were controlled by "multi_zgvt", a TopSpin program that runs a set of experiments at predetermined temperatures. Cooling was provided by a BCU-5 cooling unit and warming via the probe's built-in heaters. After the target temperature was reached the temperature was monitored until the stability of $< \pm 0.1^{\circ} \mathrm{C}$ for $>10$ mins before data were acquired. An equation was found for the calibration curve on each probe and was used to calculate temperature based on $\mathrm{KBr}$ 's chemical shift in the heating experiment (see Fig. S1).

For measuring the impact of high-power ${ }^{1} \mathrm{H}$ decoupling, a Spinal-64 block was added prior to the $90^{\circ}$ pulse on the ${ }^{79} \mathrm{Br}$ channel. This was designed to capture the maximal heating effect from the high-power decoupling by acquiring data at the end of the block where the heating should be maximal (see Fig. S2).
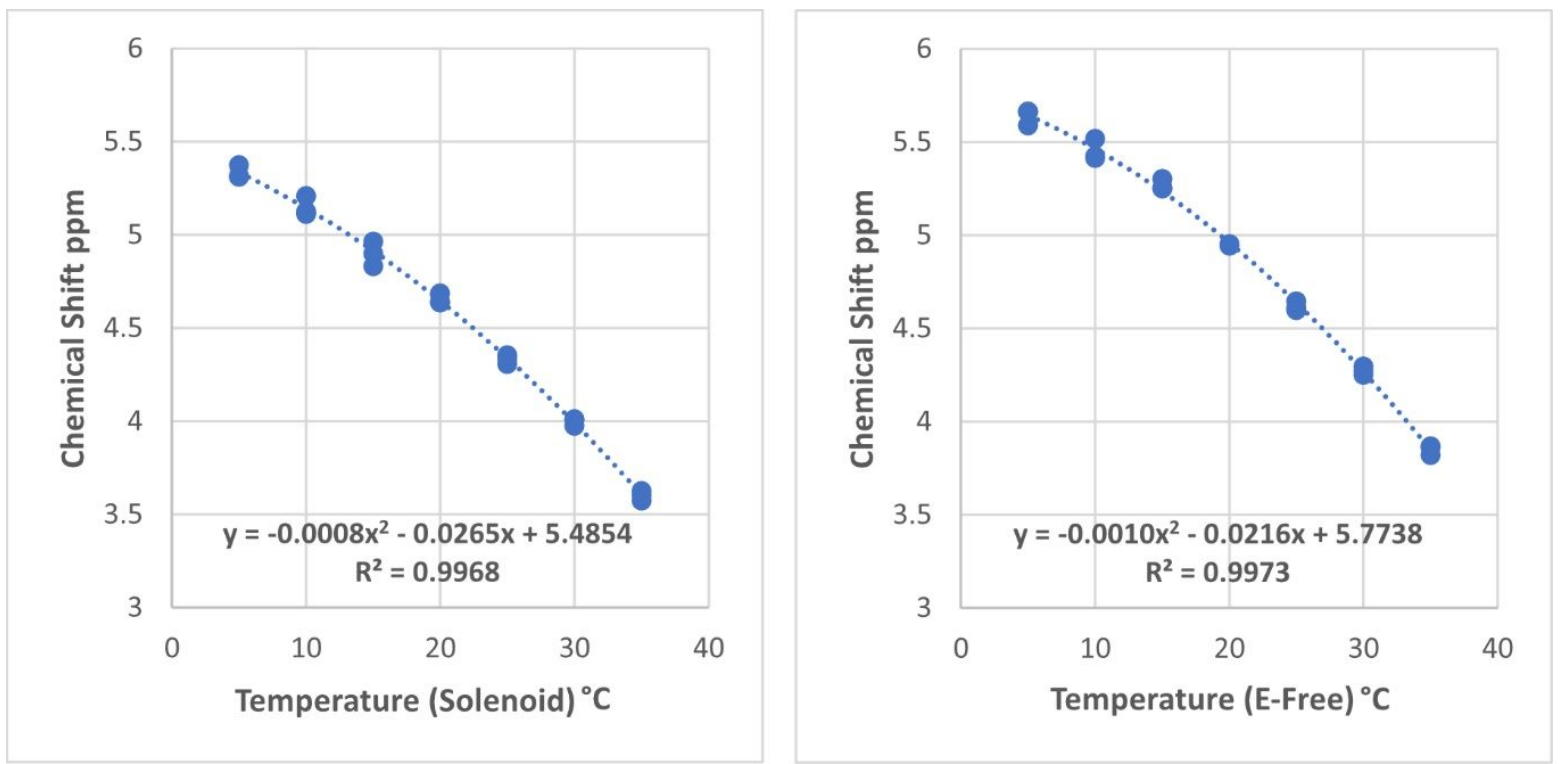

Fig. S1 Calibration curves of KBr solution chemical shifts with temperatures. The resulting equations were used to calculate sample temperature based on $\mathrm{KBr}$ chemical shift. 


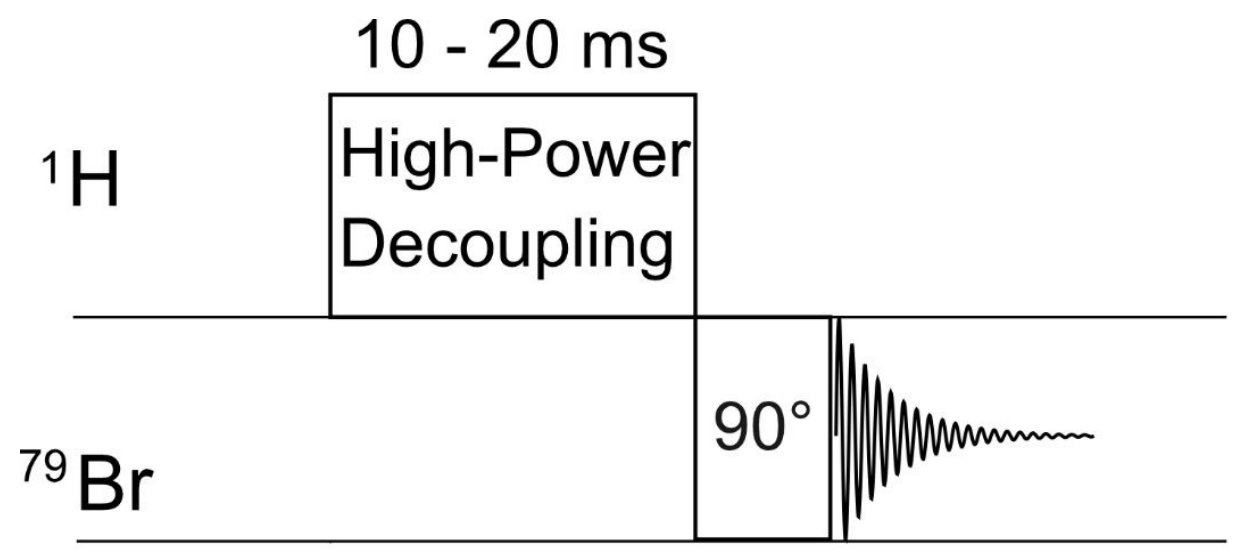

Fig. S2 Modified single-pulse sequence to capture the maximal heating caused by high-power decoupling.

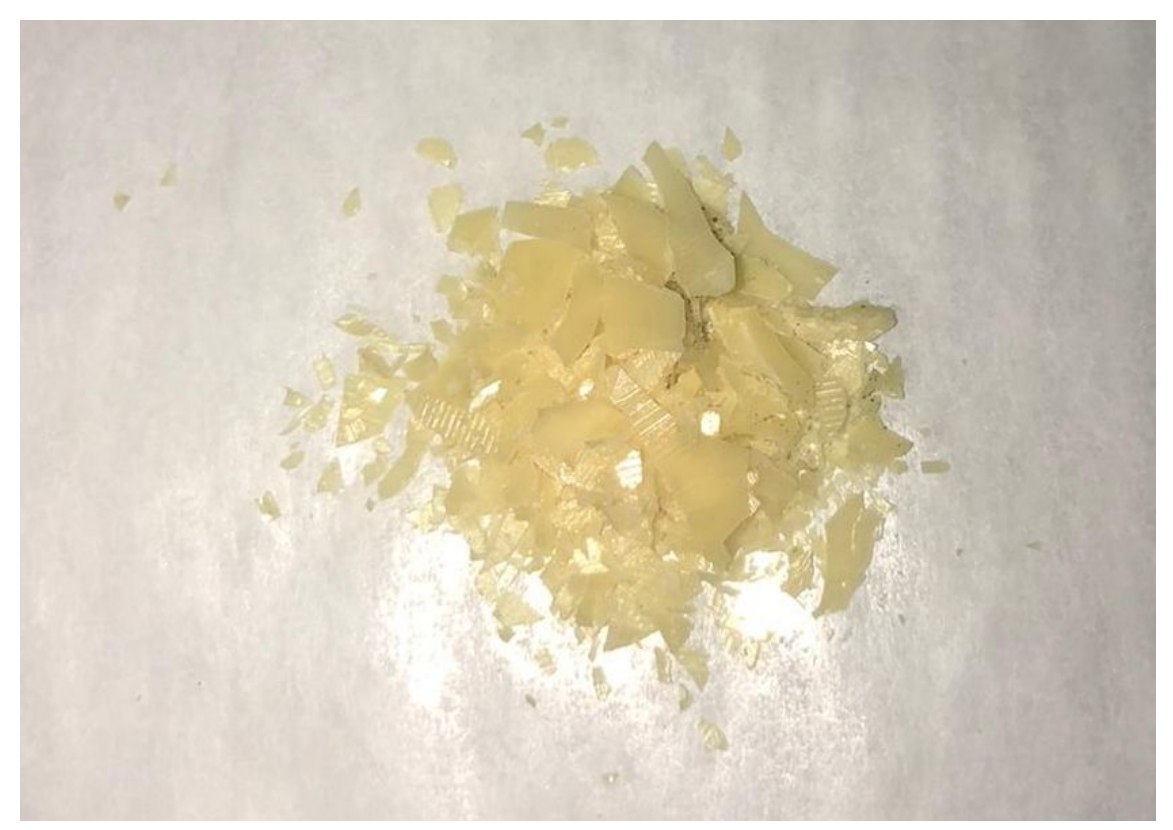

Fig. S3 Carnauba wax at room temperature. Carnauba is a leaf wax from palm trees and has a rigid crystalline structure.

\section{Reference}

(1) Mobarhan, Y. L.; Fortier-McGill, B.; Soong, R.; Maas, W. E.; Fey, M.; Monette, M.; Stronks, H. J.; 
Schmidt, S.; Heumann, H.; Norwood, W.; Simpson, A. J. Comprehensive Multiphase NMR Applied to a Living Organism. Chem. Sci. 2016, 7 (8), 4856-4866. https://doi.org/10.1039/C6SC00329J. 